\title{
El uso de antimuscarínicos en pacientes varones con síntomas del tracto urinario inferior por hiperplasia benigna de próstata y sintomas de vejiga hiperactiva
}

\author{
Ruiz Cerdá JL. \\ Servicio de Urología. Hospital Universitario La Fe de Valencia \\ Actas Urol Esp. 2006;30(9):849-855
}

$\mathrm{E}^{1}$ 1 uso de antimuscarínicos en pacientes varones con sintomas del tracto urinario inferior (STUI) por hiperplasia benigna de próstata (HBP) con sintomas de vejiga hiperactiva (VH) es un tema que está adquiriendo relevancia en los últimos años. Indicar estas drogas choca directamente contra un concepto estudiado en la Universidad: los antimuscarínicos están contraindicados en pacientes con HBP. Sin embargo, diversos factores han conducido a que este tema esté en constante revisión. La creación del síndrome de VH y toda la investigación científica llevada a cabo durante los últimos años han aportado datos epidemiológicos y fisiopatológicos que merecen la pena repasar. Sobre todo, para poder entender el porqué se ha planteado la revisión de este concepto solidamente establecido desde hace muchos años.

\section{Solapamiento de los términos sintomas del} tracto urinario inferior/vejiga hiperactiva

El término STUI, lower urinary tract symptoms (LUTS) en inglés, comprende un conjunto de síntomas de vaciado (disuria, chorro flojo, retardo, intermitencia), post miccionales (goteo terminal, sensación de vaciado incompleto) y de llenado (nocturia, frecuencia, urgencia, incontinencia de urgencia). Inicialmente, fue propuesto por Abrams en $1994^{1}$. Posteriormente, se recomendó su utilización por el panel de expertos de la $5^{\mathbf{a}}$ International Consultattion on Benign Prostatic Hyperplasia para sustituir a los términos imprecisos de "prostatismo", "hiperplasia benigna prostática clínica" o "sintomática"2. El término fue propuesto tras la observación de que los síntomas de vaciado no eran consecuencia única de la obstrucción uretral. También podian serlo por deterioro de la capacidad contráctil del detrusor. Además, se comprobó que las mujeres tenían síntomas similares sin tener patología prostática. Por lo tanto, los sintomas no necesariamente eran conse- cuencia de obstrucción, ni eran específicos de enfermedad ni tampoco exclusivos del sexo masculino. Por lo que, finalmente se llegó al término STUI-HBP para etiquetar a los pacientes con STUI por causa de HBP. En la misma reunión de expertos, se recomendó el uso del término HBP sólo cuando existiera confirmación histológica y el de aumento benigno del tamaño prostático cuando no. Desde el punto de vista funcional, y dada la ausencia de correlación entre sintomas y hallazgos urodinámicos, se recomendaba emplear el término de obstrucción sólo cuando se objetivase un flujo máximo disminuido asociado a un aumento de la presión del detrusor.

El término de VH define a un sindrome caracterizado por urgencia miccional, con o sin incontinencia de urgencia asociada, generalmente acompañado de frecuencia y nocturia. Fue propuesto por Abrams y Wein en el año 2000 y, posteriormente, recomendado por la Sociedad Internacional de Continencia ${ }^{3}$. Es decir, después del de STUI ${ }^{4}$. La creación de este término trataba de captar una realidad clínica. Ya que, sólo un tercio de los pacientes presentan incontinencia de orina asociada. Pero también, una realidad funcional, ya que no en todos los pacientes se objetiva hiperactividad del detrusor en el estudio urodinámico. De esta forma, el término hiperactividad detrusor quedó reservado para describir el hallazgo, exclusivamente urodinámico, de contracciones involuntarias durante la fase de llenado.

La creación y aplicación del término $\mathrm{VH}$ ha repercutido decisivamente en la delimitación de los espectros sintomáticos de otras patologías. Por ejemplo, la cistitis intersticial y la incontinencia urinaria femenina mixta comparten sintomas con la VH. Igualmente, los síntomas de llenado del espectro sintomático STUI-HBP son coincidentes con los de VH. Sin embargo, esta coincidencia no significa que tengan un origen fisiopatológico común. Ante 
un paciente con síntomas de llenado, es cierto que pueden ser secundarios a la HBP, pero también es cierto que pueden ser completamente independientes. Recientemente, Chapple y Roehrborn apuntaban el hecho de que en un paciente varón los síntomas de VH son síntomas de llenado del tracto urinario inferior que pueden coexistir con la HBP, el aumento del tamaño prostático o con obstrucción. Todo ello sin ser causados por patología prostática ${ }^{5}$. Como en el caso de una VH idiopática asociada, $\mathrm{VH}$ por envejecimiento propio de la edad, o por $\mathrm{VH}$ secundaria a lesiones neurogénicas no objetivables. Es evidente que, el reto clínico para orientar adecuadamente el tratamiento estriba en saber ante que situación nos encontramos y, si existen diversos factores solapados, cual es el predominante.

\section{Prevalencia de VH en varones}

Contrariamente a lo que se creía, estudios recientes han puesto de manifiesto que los sintomas de $\mathrm{VH}$ son prácticamente igual de prevalentes en varones y en mujeres. Milsom et al. en un estudio europeo sobre prevalencia de síntomas de $\mathrm{VH}$ observaron que un $16,6 \%$ de la población general de 40 años o mayores los presentaban ${ }^{6}$. Por sexos, la prevalencia en mujeres era del $17,4 \%$ y la de los hombres del 15,6\%. Estos datos fueron muy similares a los obtenidos en el estudio NOBLE realizado en USA sobre población general superior a 18 años ${ }^{7}$. Si bien, estas diferencias han aumentado en un estudio reciente utilizando la definición de $\mathrm{VH}$ actualiza$\mathrm{da}^{8}$. En ambos sexos la prevalencia aumenta con la edad. En los hombres, especialmente a partir de los 64 años. Cuando también es más prevalente la HBP.

\section{Importancia de los sintomas de llenado en varones con HBP}

La severidad de los sintomas y la repercusión sobre la calidad de vida indican la necesidad que siente el paciente de ser tratado. La disuria, el retardo, el flujo miccional débil son síntomas que el paciente sufre en silencio. Por lo general, se toleran bien hasta que llegan a un grado severo. Sin embargo, el aumento de frecuencia, la urgencia, la nocturia y la incontinencia urinaria le afectan de forma importante su vida de relación social. El impacto sobre la calidad de vida de los sintomas de llenado es mucho mayor que los de vaciado. En la actualidad, aunque los sintomas de vaciado son más prevalentes, los síntomas de llenado son el motivo por el cual los pacientes acuden con mayor frecuencia al urólogo ${ }^{8}$.
Mecanismo de acción de los fármacos para el tratamiento de la STUI-HBP y sintomas de VH

Actualmente, el mecanismo de acción de los fármacos en el tratamiento de pacientes con STUIHBP y VH asociada esta dirigido a reducir la resistencia uretral. Los alfa-bloqueantes actúan sobre el componente dinámico de la obstrucción. Disminuyen el tono adrenérgico del componente muscular de la próstata y cuello vesical. Los inhibidores de la 5 alfa reductasa actúan sobre el componente estático. Disminuyen la proliferación glandular y el tamaño prostático. Es decir, están dirigidos únicamente a tratar los sintomas de vaciado derivados de la obstrucción y no a los de llenado, derivados de la afectación vesical. Por otra parte, como ya se ha escrito, los más preocupantes para los pacientes. Por lo tanto, dichos fármacos sólo cubren parcialmente las expectativas de mejora de los pacientes. La idea de tratar a estos pacientes con antimuscarínicos, solos o junto a alfa-bloqueantes, se basa en la fisiología de los receptores adrenérgicos y muscarínicos. Puesto que la inhibición de cada uno podría ser más efectiva y beneficiosa que la terapia aislada. De esta forma, una terapia combinada abordaría los dos componentes que provocan los síntomas.

\section{Relación entre obstrucción por HBP e hiperactividad del detrusor}

En la actualidad, el abordaje terapéutico de los pacientes con STUI-HBP se lleva a cabo bajo dos supuestos: que los síntomas de llenado pertenecen al espectro sintomático de la STUI-HBP y no a otras posibles etiologías y que, si existe hiperactividad, ésta es secundaria a la obstrucción. Por lo tanto, ambas deberían mejorar con la reducción de la obstrucción. Sin embargo, la correlación entre la presencia de HBP, obstrucción y STUI es todavía controvertida. Mientras que los sintomas de vaciado y post-miccionales se justifican claramente por el efecto obstructivo sobre el tracto de salida que ejercen el aumento del tamaño prostático y el tono del músculo liso del estroma prostático, la relación entre obstrucción y los sintomas de llenado no esta tan clara. A pesar de la mejora en el entendimiento de la fisiología y farmacología del detrusor, no existe todavía una explicación satisfactoria sobre la fisiopatologia de la hiperactividad vesical. Diversos hallazgos explican la relación de causalidad entre obstrucción y desarrollo de hiperactividad. La hipertrofia de pared vesical secundaria al sobreesfuerzo que supone la obstrucción lleva asociado una 
denervación progresiva, la cual jugaría un papel esencial en los sintomas de llenado y en la hiperactividad del detrusor ${ }^{10}$. También se han encontrado alteraciones ultraestructurales en las proteínas del citoesqueleto, en la función mitocondrial y en la matriz extracelular que podrían estar implicados en la lesión del plexo periférico miovesical ${ }^{11}$. Además se ha postulado que, las áreas del detrusor donde se concentra la denervación podrían desarrollar una hipersensibilidad a la acetilcolina que predispondría a la hiperactividad vesical, junto a la liberación incontrolada de acetilcolina desde el plexo neuronal dañado. Sin embargo, siguen habiendo grandes interrogantes ${ }^{11}$. Principalmente porque, se ha puesto de manifiesto que la desobstrucción no conduce necesariamente a la desaparición de la hiperactividad $^{12}$. Aunque, tras la realización de prostatectomía, los sintomas de hiperactividad mejoran y hasta un $62 \%$ de los pacientes muestran una estabilización de la hiperactividad en la cistomanometría postoperatoria, en un 20\% no desaparece. Incluso, en estudios recientes con seguimientos a largo plazo, se ha comprobado una reaparición en todos ellos ${ }^{12}$.

Por otra parte, existe una falta de asociación entre procesos, incluso más obstructivos que la HBP, como la estenosis de uretra, la esclerosis de celda prostática, las valvas uretrales o la estenosis de meato con la presencia de hiperactividad. Por lo que, el desarrollo de hiperactividad podría ser consecuencia de, más que por el efecto obstructivo prostático, por el efecto irritativo que sobre el trígono y base de la vejiga pueda ejercer el aumento del tamaño prostático. Hay que tener en cuenta que, el trígono es una zona especialmente rica en terminaciones nerviosas. La morfología que la próstata adquiera en su crecimiento, más que el tamaño que alcance, podría relacionarse con el desarrollo de hiperactividad. En este sentido, hay ejemplos clínicos que ilustran como la obstrucción prostática no se asocia a hiperactividad. Los llamados en el pasado "prostatismos silentes" se caracterizan por ser pacientes con próstatas pequeñas pero muy obstructivas que debutan con retención aguda de orina sin ocasionar ningún síntoma de llenado.

\section{¿Por qué no se utilizan antimuscarinicos en pacientes con STUI-HBP y sintomas de VH?}

Los antimuscarínicos disminuyen la contracción del detrusor y aumentan el riesgo de retención urinaria. En pacientes con resistencia uretral aumentada este riesgo puede ser mayor. Sin embargo, de acuerdo a los conocimientos que disponemos sobre el mecanismo de acción de los antimuscarínicos, no existe una explicación clara para el aumento del riesgo de retención. Para que se produzca la contracción del detrusor se requiere acetil colina. La liberación de este neurotransmisor en los terminales postgangliónicos de los nervios parasimpáticos pélvicos estimula los receptores muscarínicos del detrusor y desencadena la contracción. Los antimuscarínicos son antagonistas competitivos de los receptores muscarínicos del detrusor y actúan bloqueando el estimulo colinérgico. Su efecto resultante es una disminución de la contractilidad del detrusor. En pacientes con $\mathrm{VH}$ el efecto terapéutico de los antimuscarínicos se produce principalmente durante la fase de llenado del ciclo miccional. Puesto que, disminuyen la sensación de urgencia miccional, retrasan la micción y reducen, en mayor o menor grado, las contracciones involuntarias del detrusor. Durante la fase de vaciado, su papel como depresores de la capacidad contráctil del detrusor parece ser muy limitado. La explicación se encuentra en el hecho de que, la contracción del detrusor durante la micción se produce como consecuencia de una masiva liberación de acetil-colina en los terminales parasimpáticos. Por su mecanismo competitivo, las concentraciones de antimuscarínicos para bloquear todos los receptores del detrusor tendrían que ser muy elevadas. Lo que requeriría dosis de antimuscarínos muy altas. Muy por encima de las dosis terapéuticas utilizadas en clínica. Por lo que, aunque el uso de antimuscarínicos a dosis muy elevadas pueda causar retención urinaria, a las dosis habituales utilizadas no provocan un deterioro de la capacidad contráctil del detrusor. Por consiguiente, el miedo a la retención urinaria no estaría justificado ${ }^{13-14}$.

\section{¿Qué evidencia científica existe publicada sobre el papel de los antimuscarinicos a dosis terapéuticas en el tratamiento de pacientes con STUI-HBP y sintomas de VH?}

La evidencia científica publicada sobre el uso de antimuscarínicos, solos o en combinación con alfabloqueantes, en pacientes con STUI- HBP y sintomas de VH es escasa. Recientemente, ha sido revisada por Novara et al. con el objetivo de clarificar si los datos disponibles hasta el momento sugieren que pudieran tener un papel en el tratamiento de estos pacientes ${ }^{15}$. Los aspectos revisados han sido la seguridad, la eficacia terapéutica y si se deben 
dar solos o combinados con alfa-bloqueantes. Hasta el momento sólo se han publicado cuatro trabajos con diseño aleatorizado y controlado y dos series de casos prospectivos ${ }^{16-21}$. El resto lo constituyen unas pocas comunicaciones a congresos científicos, editoriales y revisiones. Los estudios presentan gran variedad de metodología y de criterio. La duración del tratamiento es, por regla general, muy corta entre 1 y 3 meses con apenas seguimiento a largo plazo. Los antimuscarínicos utilizados han sido la tolterodina y la propiverina. Esta última no comercializada en España. Los alfa-bloqueantes utilizados han sido la tamsulosina, la doxazosina y la terazosina. El diseño de todos los estudios prospectivos, excepto en uno, ha sido la comparación de un grupo de pacientes tratados con alfa-bloqueante con otro grupo de pacientes tratados con una combinación de alfabloqueante y antimuscarínico. En un solo estudio el objetivo fue la seguridad, por lo que, se comparó a un grupo de pacientes tratados con placebo frente a otro con tolterodina. Todos los pacientes presentaban obstrucción demostrada. En la mayoría de las series de casos prospectivos publicadas, el uso del antimuscarínico se ha planteado tras el fracaso terapéutico con alfa-bloqueante. De tal forma que, a los pacientes se les reemplazó el alfabloqueante por el antimuscarínico o bien se les añadió en terapia combinada.

Con relación a la seguridad, la toma de antimuscarínicos no se asocia a un aumento en la tasa de efectos adversos. Aunque, si se aprecia un aumento del volumen residual, éste no es clínicamente significativo ni motivo para retirar el fármaco. Tampoco se observa un aumento de la tasa de retención aguda de orina. Estos datos sobre la baja probabilidad de retención urinaria ya habían sido adelantados, de forma indirecta, en los metaanálisis sobre la eficacia terapéutica de los antimuscarínicos frente a

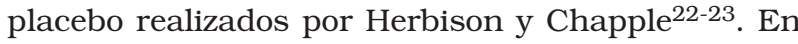
estas revisiones se concluyó que, aunque la mayoría de estudios no aportaban datos específicos sobre los efectos secundarios, en el grupo de pacientes tratados no se observó un aumento, ni clínica ni estadísticamente significativa en el volumen residual, en la máxima capacidad vesical o en la tasa de retención urinaria frente al grupo placebo. Únicamente la oxibutinina demostró un aumento significativo del riesgo de retención urinaria con respecto al placebo en el metaanálisis llevado a cabo por Chapple. Por lo que, estos hallazgos clínicos de bajo riesgo de retención urinaria, se correlacionan con lo expuesto anteriormente en lo que respecta al efecto de los antimuscarínicos.

Con respecto a la eficacia, en la mayoría de estudios, la administración de antimuscarínicos consiguió mejorar significativamente la sintomatología y la calidad de vida. La frecuencia miccional nocturna fue el síntoma que más se redujo. En cuanto a los hallazgos urodinámicos, se produjo una reducción de la presión máxima del detrusor durante la micción y durante la fase de llenado.

De acuerdo a las conclusiones de los autores, los datos disponibles en este momento son alentadores en cuanto a la seguridad y la eficacia. Sin embargo, la evidencia existente procede de un limitado número de ensayos clínicos aleatorizados y controlados de bajo nivel de calidad de evidencia. Ya que, sólo un trabajo se clasificó como de buena calidad de acuerdo a los criterios de medicina basada en la evidencia. El resto presentaban sesgos metodológicos importantes. Por lo que concluyen que, la evidencia no es suficiente para soportar el uso clínico de antimuscarínicos, en combinación con alfa-bloqueantes o solos para pacientes con HBP y sintomas de VH asociados. Los autores acaban con la manida frase "mayor número de ensayos controlados y aleatorizados, bien diseñados y con seguimiento a largo plazo son necesarios" 14,24

\section{Aplicación en la práctica clínica de la evidencia}

Uno de los aspectos más importantes a la hora de plantear la aplicación de la evidencia a la práctica clínica es su validez externa. Hay que tener en cuenta que, en prácticamente la totalidad de estudios disponibles, el criterio de inclusión de los pacientes ha sido la demostración urodinámica de hiperactividad y de obstrucción. Por lo tanto, esta evidencia sólo sería aplicable a pacientes en los que se disponga de un estudio urodinámico previo. Este requisito plantea un nuevo reto para su aplicación en la práctica diaria. Puesto que, quedaría por definir el ámbito de aplicación de este tipo de tratamiento (asistencia primaria versus especializada), qué tipo de pruebas diagnósticas serían necesarias y su secuencia de utilización (cuestionario de síntomas, tacto rectal, determinación del antígeno específico prostático, flujometría, medición de residuo postmiccional, ecografía vesico-prostática, estudio de presión y flujo) y, por último, establecer un algoritmo terapéutico que defina bien los criterios sin- 
tomáticos y funcionales de los pacientes que más se puedan beneficiar del uso de antimuscarínicos, en régimen de monoterapia o combinados con alfa-bloqueantes.

\section{¿Qué hacer?}

Puestas así las cosas, ¿la ausencia de evidencia científica invalida el uso de antimuscarínicos en estos pacientes? En otras palabras, ¿es posible seguir tomando decisiones individuales aunque no tengamos este soporte científico? Yo creo sinceramente que, en este caso, la respuesta es afirmativa. En este sentido me gustaría realizar una serie de comentarios. Hay que tener en cuenta que la medicina basada en la evidencia científica no es sólo la evidencia publicada. La definición exacta de medicina basada en la evidencia es la "integración de la maestría clínica individual con la mejor evidencia científica disponible. Todo ello, además, aplicado con sentido común y teniendo en cuenta las preferencias de los pacientes". En muchas ocasiones, se olvida que la práctica de la medicina basada en la evidencia se fundamenta también en la maestría clínica y que la opinión individual también forma parte de ella. Es posible que no exista evidencia publicada, pero no quiere decir que la experiencia procedente de la observación individual sea inválida. Por lo menos, es complementaria. Como bien, queda reflejada en la definición expuesta. A la hora de resolver el problema clínico que nos ocupa, el médico necesita de respuestas inmediatas y debe utilizar su maestría clínica. No puede esperar a disponer de evidencia científica.

Pero, es evidente que nos enfrentamos a un problema ético. A este respecto, recientemente Madersbacher ${ }^{25}$ ha puntualizado que, un tratamiento empírico se puede considerar justificado cuando los sintomas son preocupantes y tienen impacto sobre la calidad de vida, cuando el tratamiento tiene baja morbilidad y cuando está prevista o se plantea un cuidadoso y precoz seguimiento y valoración de la eficacia terapéutica. El caso que nos ocupa cumple todos estos criterios. Los síntomas de llenado son los más preocupantes para el enfermo y los que más afectan a su calidad de vida. En cuanto a la morbilidad, no se trata del uso de fármacos nuevos en los que se desconoce si se pone en peligro la seguridad del paciente. Todo lo contrario, existe una vasta experiencia en este sentido. El mayor riesgo es la probable retención crónica o aguda que no compromete la vida del paciente, si se tiene un seguimiento estrecho y cuidadoso. Además, es totalmente reversible. De lo que se trata es de valorar su eficacia para poder abrir una nueva indicación terapéutica.

Llegado hasta aquí, ¿cómo actuar ante un paciente varón con STUI-HBP y síntomas de VH? Desde mi punto de vista (maestría clinica), hay dos aspectos fundamentales para contestar a esta pregunta que son: el espectro sintomático del paciente y las exploraciones complementarias de que disponemos en nuestro ámbito de trabajo para un diagnóstico funcional correcto del tracto urinario inferior. El espectro sintomático de pacientes con STUIHBP es amplio. Existen pacientes en los que predominan los síntomas que indican la existencia de determinado grado de obstrucción y pacientes en los que predominan los síntomas que indican alteración de la fase de llenado. En mi opinión, la presencia de urgencia, con o sin incontinencia, es el sintoma clave que permite clasificar a los pacientes. Ya que hace sospechar que el paciente puede tener una $\mathrm{VH}$ asociada. $\mathrm{El}$ aumento de frecuencia miccional, aún siendo un síntoma de llenado, es poco específico. Puesto que, está presente en prácticamente todos los pacientes. El aumento de la frecuencia miccional sí es útil cuando se presenta como sintoma principal y predominantemente nocturno. Ya que es característico de la poliuria nocturna. Enfermedad que no está relacionada ni con la vejiga ni con la próstata y es fácilmente diagnosticable mediante un diario de frecuencia - volumen. De acuerdo a esta premisa, se puede clasificar a los pacientes en tres grupos: pacientes con sintomas de predominio obstructivo sin urgencia, pacientes en los que el sintoma principal es la urgencia sin síntomas obstructivos $\mathrm{y}$, por último, el grupo de pacientes con síntomas obstructivos y urgencia.

Es evidente que, para el primer grupo el tratamiento debe ir dirigido hacia los síntomas obstructivos. Normalmente, no se plantea el uso de antimuscarínicos. Para el segundo, lo más importante es pensar en su existencia. Por esto, es esencial que se trasmita la información obtenida de los estudios epidemiológicos de que la $\mathrm{VH}$ es igual de prevalente en hombres como en mujeres. De este modo, mejoraría el enfoque diagnóstico de estos pacientes limitado por el prejuicio de que, la causa de los STUI en el hombre es la próstata hasta que no se demuestre lo contrario. Este grupo de pacientes no presentan sintomas obstructivos. Sin embargo, son los que 
con mayor frecuencia se encuentran en la práctica clínica tratados con medicación dirigida a la próstata. Cuando, por otra parte, son los que más se van a beneficiar de los antimuscarínicos.

Por último, el manejo del tercer grupo de pacientes es el que requiere mayor maestría clínica. Es evidente que, aunque ya se ha comentado que los antimuscarínicos son seguros en estos pacientes, el punto esencial para su enfoque es cuantificar el componente obstructivo. Porque, independientemente de la presencia de urgencia, si el paciente tiene obstrucción habrá que tratarla, médica o quirúrgicamente. $\mathrm{Y}$ aquí es donde entra en juego el segundo aspecto en el manejo de estos pacientes: las exploraciones complementarias de que disponemos en nuestro ámbito de trabajo para un diagnóstico funcional correcto. Hay que tener en cuenta que, la correlación entre síntomas, tamaño prostático y obstrucción no es perfecta. Además, el diagnóstico de obstrucción sólo es posible si se realiza un estudio de presión y flujo. Estos dos factores hacen que, el abordaje diagnóstico necesariamente tenga que ser múltiple y escalonado y que, la probabilidad de error diagnóstico y terapéutico será menor cuanto mayor sea el número de pruebas complementarias. Por lo que, todas las decisiones terapéuticas llevaran implícitas un riesgo (probabilidad de retención), mayor o menor, que habrá que asumir y tratar de minimizar de acuerdo a la maestría clínica individual de cada médico. Ya que, la mayor seguridad sólo la tendremos cuando conozcamos la alteración funcional subyacente de un paciente determinado. Esto sólo es posible realizando una cistomanometría de llenado, para objetivar la hiperactividad del detrusor, y una cistomanometría de vaciado o presión y flujo, para objetivar y cuantificar el grado de obstrucción.

De acuerdo a la disponibilidad e invasividad de las pruebas diagnósticas se establecen tres ámbitos de trabajo. El primer ámbito es en el trabajan la mayoría de urólogos españoles. En él, se toman decisiones con la información que aportan las herramientas únicamente disponibles en la consulta. Es decir, historia clínica, evaluación de síntomas, tacto rectal y antígeno específico prostático ${ }^{26}$. La información que aportan estas pruebas sobre el grado de obstrucción es escasa. Sin embargo, la edad avanzada por encima de los 70 años, el predominio de la urgencia sobre los síntomas obstructivos, la palpación de una próstata plana o poco aumentada y un PSA inferior a $2 \mathrm{ng} / \mathrm{ml}$ son indica- dores de que la $\mathrm{VH}$ predomina sobre la obstrucción y que, en este grupo de pacientes, la asociación de un antimuscarínico puede ser beneficiosa ${ }^{27}$. En el segundo ámbito trabajan un porcentaje de urólogos de ambulatorio y la mayoría de urólogos hospitalarios. En él se toman decisiones con la información procedente de pruebas morfológicas y urodinámicas no invasivas. Es decir, ecografía vesico-prostática con estudio de tamaño prostático y medición de residuo post-miccional y flujometría. La información que aportan estas pruebas sobre el grado de obstrucción no es suficientemente exacta. Si bien permiten descartar obstrucción más que afirmar. Un Qmax superior a $15 \mathrm{ml} / \mathrm{seg}$ y ausencia de residuo postmiccional descartan con elevada probabilidad la obstrucción. Por el contrario, un Qmax inferior a 10 $\mathrm{ml}$ y un elevado residuo postmiccional superior al $40 \%$ de la máxima capacidad vesical indican obstrucción con elevada probabilidad. Por último, en el tercer ámbito trabajan aquellos urólogos que pueden disponer de aparatos de urodinámica para poder llevar a cabo un estudio funcional completo del tracto urinario inferior. La cistomanometría de llenado informa de la presencia de hiperactividad del detrusor y cuantifica su severidad. La cistomanometría de vaciado informa de la presencia de obstrucción y, mediante los nomogramas de presión y flujo, cuantifica su severidad y el grado de contractilidad del detrusor. Disponiendo de esta información funcional, la prescripción de antimuscarínicos se realiza dirigida y se puede estimar el riesgo de retención.

Resumiendo podemos decir que, de acuerdo a la evidencia científica publicada la utilización de antimuscarínicos en pacientes con STUI-HB con sintomas de VH es segura. Por otra parte, es cierto que se necesita más evidencia de eficacia terapéutica. Sin embargo, son muchos los enfermos que acuden a nuestra consulta con estas características y debemos tomar decisiones individuales. A este respecto, nos puede ser de utilidad plantearnos la siguiente pregunta ¿se puede beneficiar este enfermo del uso de un antimuscarínico? La respuesta se obtiene aplicando nuestra maestría clínica.

\section{REFERENCIAS}

1. Abrams P. New words for old: lower urinary tract symptoms for "prostatism". BMJ 1994;308(6934):929-930.

2. Abrams P, Griffiths D, Hofner K. The urodynamic assessment of lower urinary tract symptoms (committee 7). In Chatelain C, Denis L, Foo KT, Khoury S, Mc Connell J, editors. Benig Prostatic Hyperplasia. 5th Internacional Consultation on BPH. 2000; p. 227-282. 
3. Abrams P, Wein AJ. Introduction: overactive bladder and its treatment. Urology. 2000 May;55(5A Suppl):1-2.

4. Abrams P, Cardozo L, Fall M, Griffiths D, Rosier P, Ulmsten $\mathrm{U}$ et al. The Standardisation of terminology of lower urinary tract function: Report from the Standardisation subcommittee of the International Continence Society. Urology. 2003 Jan;61(1):37-49.

5. Chapple CR, Roehrborn CG. A shifted paradigm for the further understanding, evaluation, and treatment of lower urinary tract symptoms in men: focus on bladder. Eur Urol. 2006 Apr;49(4):651-659.

6. Milsom I, Abrams P, Cardozo L, Roberts RG, Thüroff J, Wein AJ. How widespread are the symptoms of an overactive bladder and how are they managed? A populationbased prevalence study. BJU International. 2001 Jun;87 (9):760-766.

7. Stewart WF, Van Rooyen JB, Cundiff GW, Abrams P, Herzog AR, Corey R et al. Prevalence and burden of overactive bladder in the United States. World J Urol. 2003 May;20(6):327-336.

8. Irwin DE, Milsom I, Reilly K, Hunskaar S, Kopp Z, Herschorn $\mathrm{S}$ et al. Prevalence of overactive bladder syndrome: European results from the EPIC study. Eur Urol. Suppl 2006;5(2):115.

9. Peters TJ, Donovan JL, Kay HE, Abrams P, de la Rosette JJMCH, Porru D et al. The International Continence Society " benig prostatic hyperplasia" study: the botherssomeness of urinary symptoms. J Urol. 1997 Mar;157(3): 885-889.

10. Gosling A, Kung LS, Dixon S, Horan P, Whitbeek C, Levin RM. Correlation between the structure and function of the rabbit urinary bladder following partial outlet obstruction. J Urol. 2000 Apr; 163(4):1349-1356.

11. Andersson KE. Storage and voiding symptoms: pathophysiologic aspects. Urology. 2003 Nov;62(5 Suppl 2):3-10.

12. Thomas AW, Cannon A, Bartlett E, Jones J, Abrams P. The natural history of lower urinary tract dysfunction in men: minimum 10-year urodynamyc follow-up of untreated bladder obstruction. BJU Int. 2005 Dec;96(9):1301-1306.

13. Andersson KE, Yoshida M. Antimuscarinics and the over active detrusor - which is the main mechanism of action? Eur Urol. 2003 Jan;43(1): 1-5.

14. Reynard JM. Does anticholinergic medication have a role for men with lower urinary tract symptoms / benig prostatic hyperplasia either alone or in combination with other agents? Curr Opin Urol. 2004 Jan; 14(1):13-16.

15. Novara G, Galfano A, Ficarra V, Artibani W. Anticholinergic durgs in patients qith bladder outlet obstruction and lower urinary tract symptoms: a systematic review. Eur Urol. 2006 Oct;50(4):675-683.

16. Athanasopoulos A, Gyftopoulos K, Giannitsas K, Fisfis J, Perimenis P, Barbalias G. Combination treatment with an alfablocker plus an anticholinergic for bladder outlet obstruction: a prospective, randomized, controlled study. J Urol. 2003 Jun;169(6):2253-2256.

17. Lee KS, Choo MS, Kim DY, Kim JC, Kim HJ, Min KS et al. Combination treatment with propiverine hydrochloride plus doxazosin controlled release gastrointestinal therapeutic system formulation for overactive bladder and coexisting benign prostatic obstruction: a prospective, randomized, controlled multicenter study. J Urol. 2005 Oct;174(4 Pt 1):1334-1338.

18. Abrams P, Kaplan S, De Koning Gans H, Millard R. Safety and tolerability of overactive for the treatment of overactive bladder in men with bladder outlet obstruction. J Urol. 2006 Mar; 175(3 Pt 1):999-1004.

19. Saito H, Yamada T, Oshima H. A comparative study of the efficacy and safety of tamsulosin hydrochlorid alone and combination of propiverine hydrochloride and tansulosin hydrochloride in the benign prostatic hypertrophy with pollakisuria and/or urinary incontinence. Jpn J Urol Surg. 1999;12:525-536.

20. Kaplan SA, Walmsley K, Te AE. Tolterodine extended realease attenuates lower urinary tract symptoms in men with benign prostatic hyperplasia. J Urol. 2005 Dec;174(6): 2273-2275.

21. Lee JY, Kim HW, Lee SJ, Koh JS, Suh HJ, Chancellor MB. Comparison of doxazosin with or without tolterodine in men with symptomatic bladder outlet obstruction and an overactive bladder. BJU Int. 2004 Oct;94(6):817-820.

22. Herbison Meter, Hay-Smith J, Ellis G, Moore K. Effectiveness of anticholinergic drugs compared with placebo in the treatment of overactive bladder: systematic review. BMJ 2003 Apr 19;326(7394):841-844.

23. Chapple C, Khullar V, Gabril Z, Dooley JA. The effects of antimuscarinic treatments in overactive bladder: a systematic review and meta-analysis. Eur Urol. 2005 Nov;48(5): 5-26.

24. Irani J. Anticholinergic drugs in patients with bladder outlet obstruction and lower urinary tract symptoms: Where do we stand in 2006? Eur Urol. 2006 Oct;50(4):653654.

25. Madersbacher H. Overactive bladder: a clinical entity or a marketing hype? Eur Urol. 2005 Mar;47(3):273-276.

26. Carballido Rodríguez J, Badía Llach X, Gimeno Collado A, Regadera Sejas L, Dal-Re Saavedra R, Guilera Sarda M. Validez de las pruebas utilizadas en el diagnóstico inicial y su concordancia con el diagnóstico final en pacientes con sospecha de hiperplasia benigna de próstata. Actas Urol Esp. 2006;30(7):667-674.

27. Mochtar CA, Kiemeney LA, van Riemsdijk MM, Barnett GS, Laguna MP, Debruyne FM et al. Prostate-specific antigen as an estimator of prostate volume in the management of patients with symptomatic benign prostatic hyperplasia. Eur Urol. 2003 Dec;44(6):695-700.

Dr. J.L. Ruiz Cerdá

Servicio de Urología. Hospital Universitario La Fe

Avda. Campanar, 21

46009 Valencia

E-mail: jose.1.ruiz@uv.es 